\title{
Fused heterocycles 11. Synthesis of tricyclic fused pyrazolines by the reaction of 3-arylidenechromanones and 3-arylidene-1-thiochromanones with hydrazine
}

\author{
Albert Lévai, *a Katalin E. Kövér, ${ }^{\text {b }}$ and József Jekőc \\ ${ }^{a}$ Department of Organic Chemistry, University of Debrecen, P.O.Box 20, \\ H-4010 Debrecen, Hungary, \\ ${ }^{b}$ Department of Inorganic and Analytical Chemistry, University of Debrecen, \\ H-4010 Debrecen, Hungary, \\ ${ }^{c}$ Department of Chemistry, College of Nyíregyháza, Sóstói u. 31/b, H-4400 Nyíregyháza, \\ Hungary \\ E-mail: alevai@puma.unideb.hu
}

Dedicated to Professor Dr. Waldemar Adam on the occasion of his 70th birthday

\begin{abstract}
New tricyclic fused pyrazolines 8-21 have been synthesized by the reaction of 3arylidenechromanones 1-4 and 3-arylidene-1-thiochromanones 5-7 with hydrazine in hot acetic acid or propionic acid solution. Structures of all new compounds have been elucidated by microanalyses, ${ }^{1} \mathrm{H}$ - and ${ }^{13} \mathrm{C}-\mathrm{NMR}$, IR and mass spectroscopic measurements.
\end{abstract}

Keywords: 3-Arylidenechromanones, 3-arylidene-1-thiochromanones, hydrazine, pyrazolines

\section{Introduction}

Pyrazolines are important nitrogen-containing heterocyclic compounds. Owing to their useful bioactivities, ${ }^{2-4}$ increasing attention has been focused on this ring system. 2-Pyrazolines seem to be the most frequently studied pyrazoline type compounds. As a consequence, various methods have been used for their synthesis and their numerous representatives have been described in the literature. ${ }^{5-7}$ An especially popular procedure is based on the reaction of $\alpha, \beta$-unsaturated ketones with hydrazines to afford 2-pyrazolines. ${ }^{6,7}$ Synthesis of tricyclic fused pyrazolines by the reaction of exocyclic $\alpha, \beta$-unsaturated ketones and hydrazines has also been studied by several research groups. ${ }^{1,8-25}$ As a continuation of our activity on the investigation of the reaction of 
exocyclic $\alpha, \beta$-unsaturated ketones with hydrazines, ${ }^{1,12,17,18,21,22}$ in this paper we report on the synthesis of new tricyclic fused pyrazolines by the reaction of 3-arylidenechromanones and 3arylidene-1-thiochromanones with hydrazine in hot acetic acid or propionic acid solution.

\section{Results and Discussion}

Formerly we have worked out a simple and convenient procedure for the synthesis of E-3arylidenechromanones and Z-3-arylidene-1-thiochromanones by the piperidine-catalyzed condensation of chromanone and 1-thiochromanone with aromatic aldehydes. ${ }^{26,27}$ This easy access of these exocyclic $\alpha, \beta$-unsaturated ketones made possible for us to study their various chemical transformations. ${ }^{28}$ Among others, spiro-1-pyrazolines were synthesized by their treatment with diazomethane. ${ }^{29,30}$ Their reaction with phenylhydrazine in hot pyridine provided trans-2,3,3a,4-tetrahydro-3-aryl-2-phenyl[1]benzopyrano[4,3-c]pyrazoles and trans-2,3,3a,4tetrahydro-3-aryl-2-phenyl[1]benzothiopyrano[4,3-c]pyrazoles in diastereoselective reaction. ${ }^{21}$ However, if 3-arylidenechromanones and 3-arylidene-1-thiochromanones were reacted with hydrazine hydrate in hot pyridine, an intractable multicomponent reaction mixture was obtained in each case. For this reason, these reaction conditions appear to be inadequate for this purpose. If methanol or ethanol were used as solvents, as in the case of some other exocyclic $\alpha, \beta$ unsaturated ketones, ${ }^{9}$ the progress of the reaction was too slow which, at the same time, gave rise for undesirable decompositions as well. To overcome these disadvantages, our aim was to find adequate reaction conditions for the preparation of the planned tricyclic fused pyrazolines.

Sangwan et al. ${ }^{10,15}$ synthesized tricyclic fused pyrazolines by the reaction of various exocyclic $\alpha, \beta$-unsaturated ketones, including the 3-benzylidenechromanone (1) as one of our starting materials, and hydrazine hydrate in hot acetic acid. Under these reaction conditions diaseteromeric mixtures of $\mathrm{N}$-acetylated 3,3a-trans- and 3,3a-cis-isomers were obtained. In their study, the trans-cis stereochemistry was determined by ${ }^{1} \mathrm{H}-\mathrm{NMR}$ spectroscopy by using the chemical shift values of the aliphatic 3,3a-protons. These results prompted us to try to use acetic acid and propionic acid as solvents in our experiments.

3-Arylidenechromanones 1-4 and 3-arylidene-1-thiochromanones 5-7 were allowed to react with hydrazine hydrate in hot acetic acid or in hot propionic acid and diastereomeric mixtures ( $c f$. Table 1) of tricyclic fused pyrazolines 8-21 (Scheme 1) were obatianed in good yields (72-87\%). The diastereomeric ratio of compounds 8-21 was determined by ${ }^{1} \mathrm{H}-\mathrm{NMR}$ spectroscopy and the trans- and cis-diastereomers were separated by silica gel column chroamtography using toluene:ethyl acetate $(4: 1 \mathrm{v} / \mathrm{v})$ as eluent. Structure and stereochemistry of each isolated isomer have been determined by a combined utilization of various spectroscopic methods. 
<smiles>[R]C1=CC/C(=C\c2ccc([X])cc2)C(=O)c2ccccc21</smiles>

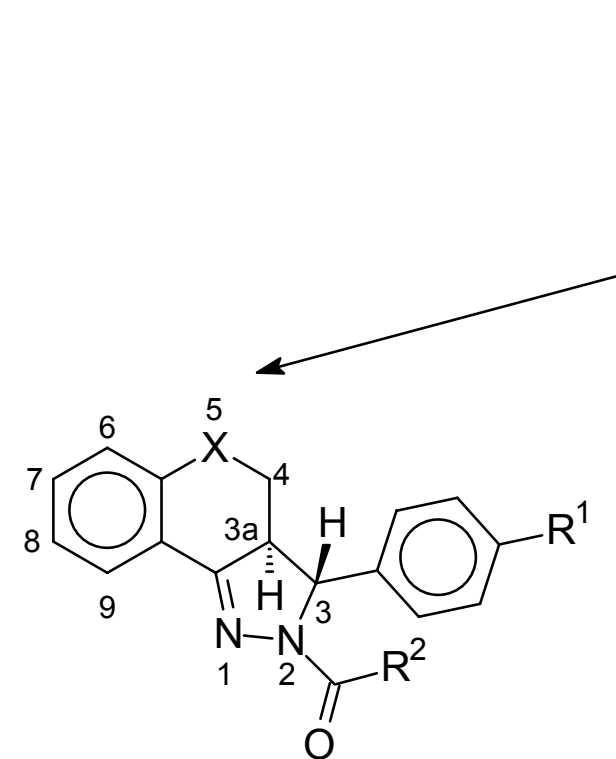

3,3a-trans-8-21

1-7

$\mathrm{H}_{2} \mathrm{NNH}_{2}$ $\mathrm{R}^{2} \mathrm{COOH}$

1, 8: $X=O, R^{1}=H, R^{2}=M e$

2, 9: $X=O, R^{1}=R^{2}=M e$

3, 10: $X=O, R^{1}=M e O, R^{2}=M e$

4, 11: $X=O, R^{1}=C l, R^{2}=M e$

1, 12: $X=O, R^{1}=H, R^{2}=E t$

2, 13: $X=O, R^{1}=M e, R^{2}=E t$

3, 14: $X=O, R^{1}=M e O, R^{2}=E t$
4, 15: $X=O, R^{1}=C l, R^{2}=E t$

5, 16: $X=S, R^{1}=H, R^{2}=M e$

6, 17: $X=S, R^{1}=R^{2}=M e$

7, 18: $X=S, R^{1}=M e O, R^{2}=M e$

5, 19: $X=S, R^{1}=H, R^{2}=E t$

6, 20: $X=S, R^{1}=M e, R^{2}=E t$

7, 21: $X=S, R^{1}=M e O, R^{2}=E t$

\section{Scheme 1}

Elemental analyses and mass spectroscopic data unambiguously proved the elemental composition of compounds 8-21. In the IR spectra a characteristic intense amide carbonyl band assigned between 1651 and $1672 \mathrm{~cm}^{-1}$ and a $\mathrm{C}=\mathrm{N}$ band between 1593 and $1614 \mathrm{~cm}^{-1}$ refer to the formation of an $\mathrm{N}$-acylated pyrazoline ring. 
Table 1. Yield and diastereomeric composition of compounds 8-21

\begin{tabular}{|c|c|c|c|}
\hline Compounds & Yield (\%) & trans-Diastereomer (\%) & cis-Diastereomer (\%) \\
\hline $\mathbf{8}$ & 75 & 50 & 50 \\
\hline $\mathbf{9}$ & 82 & 50 & 50 \\
\hline $\mathbf{1 0}$ & 77 & 50 & 50 \\
\hline $\mathbf{1 1}$ & 87 & 50 & 50 \\
\hline $\mathbf{1 2}$ & 74 & 55 & 45 \\
\hline $\mathbf{1 3}$ & 73 & 60 & 40 \\
\hline $\mathbf{1 4}$ & 75 & 55 & 45 \\
\hline $\mathbf{1 5}$ & 79 & 55 & 45 \\
\hline $\mathbf{1 6}$ & 87 & 50 & 50 \\
\hline $\mathbf{1 7}$ & 76 & 50 & 50 \\
\hline $\mathbf{1 8}$ & 74 & 50 & 50 \\
\hline $\mathbf{1 9}$ & 82 & 60 & 40 \\
\hline $\mathbf{2 0}$ & 78 & 55 & 45 \\
\hline 21 & 81 & 55 & 45 \\
\hline
\end{tabular}

In the ${ }^{1} \mathrm{H}-\mathrm{NMR}$ spectra ( $c f$. Experimental Section) both the chemical shift values and the multiplicities of the signals unambiguously reveal the characteristics of a tricyclic pyrazoline ring system. As mentioned, we have succeeded to separate the diastereomers formed which made possible a reliable determination of the relative configuration of the two centres of chirality. This cannot be achieved solely on the basis of the coupling constant values of the protons attached to the C-3 and C-3a carbon atoms. For this reason, NOE measurements of compounds cis-8 and trans $\mathbf{- 8}$ have been performed. ${ }^{31}$ The strong NOE observed between $3-\mathrm{H}$ and $3 \mathrm{a}-\mathrm{H}$ in the cis-8 (Fig. 1a) indicates the spatial proximity of the relevant atoms, correspondingly supports the cis orientation of the 3-H and 3a-H hydrogens. On contrary, the weak NOE detected between the 3$\mathrm{H}$ and $3 \mathrm{a}-\mathrm{H}$ in trans-8 (Fig. 1b) corroborates the trans arrangement of the respective atoms. The large upfield shifts of the $4-\mathrm{H}_{\mathrm{eq}}$ and $4-\mathrm{H}_{\mathrm{ax}}$ in the case of the cis-8 -0.5 and -0.9 ppm compared to the corresponding shifts of the trans-8, agrees also with the NOE-assigned relative configuration, viz. in the case of cis orientation of the $3-\mathrm{H}$ and $3 \mathrm{a}-\mathrm{H}$ in the $c i s-\mathbf{8}$ the $4-\mathrm{H}$ atoms are placed above the plane aromatic ring experiencing the strong shielding effect of the ring current. 
(a)

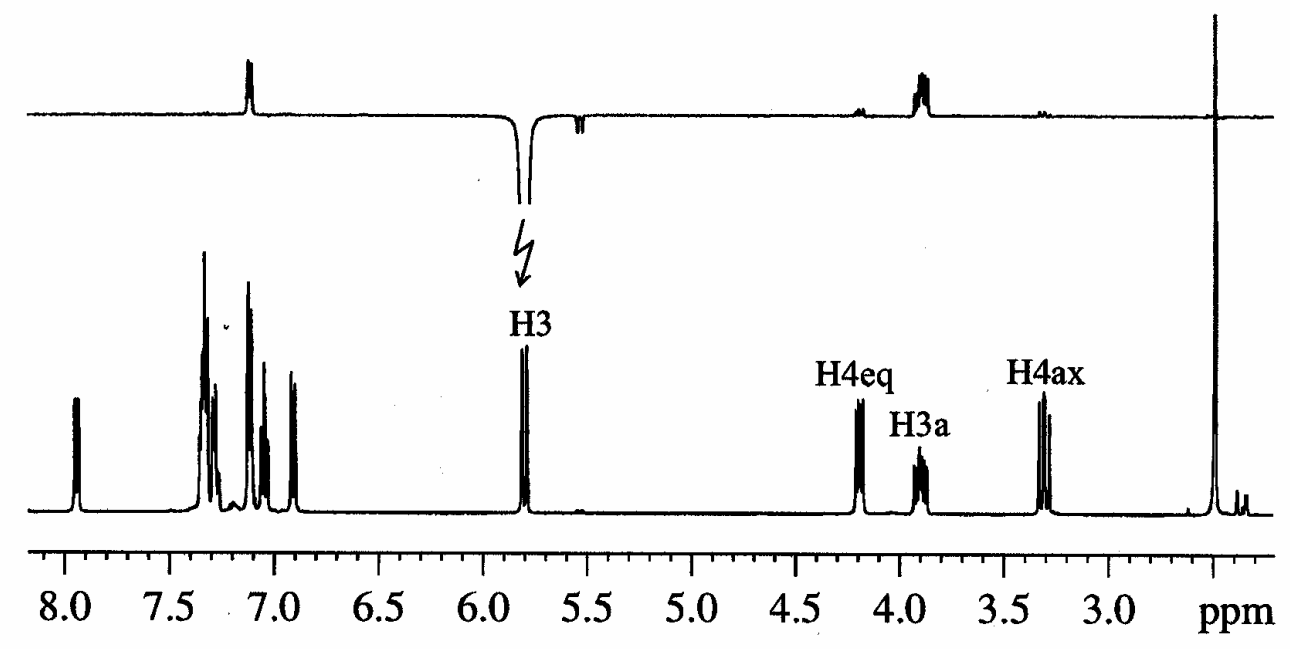

(b)

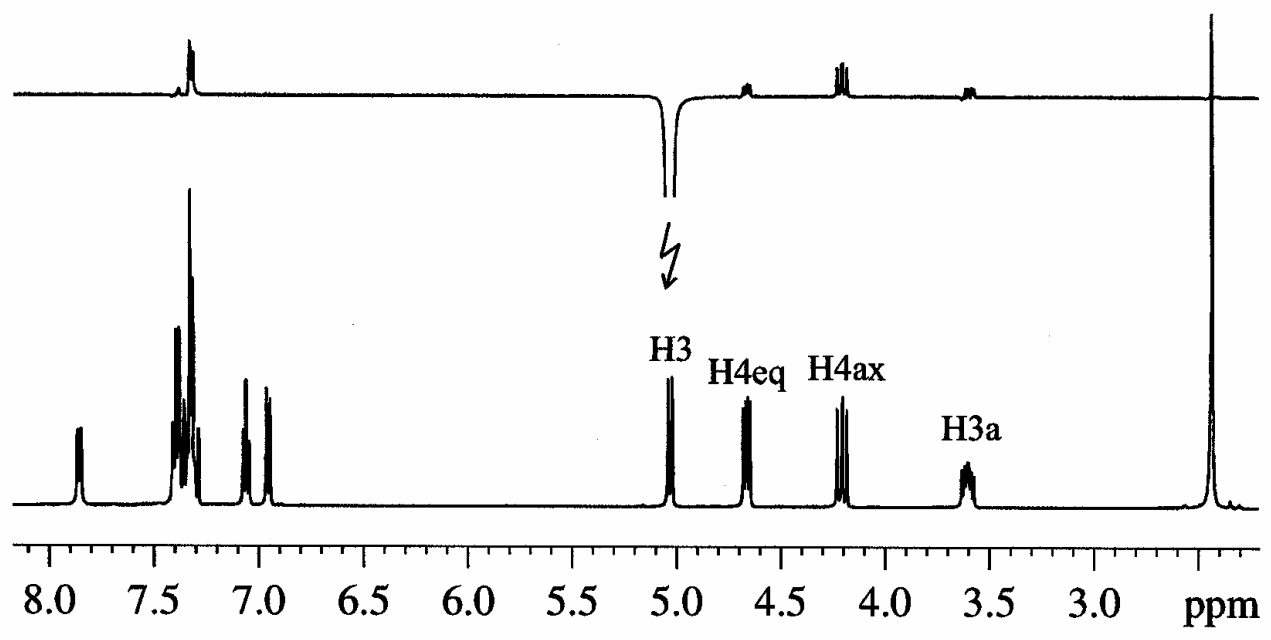

Figure 1. NOE measurements of compounds $c i s-\mathbf{8}$ and trans-8.

The presence of a tricyclic pyrazoline ring system is corroborated by the chemical shift values of the aliphatic carbon atoms in the ${ }^{13} \mathrm{C}-\mathrm{NMR}$ spectra ( $c f$. Experimental Section). The 
chemical shifts of the carbon atoms of the acetyl and the propionyl groups also confirm the $\mathrm{N}$ acylated character of the isolated pyrazolines.

On the basis of the literature data on the synthesis of 2-pyrazolines by the reaction of $\alpha, \beta-$ unsaturated ketones with hydrazines under acidic conditions, ${ }^{32-34}$ we assume a plausible mechanism (Scheme 2) for the formation of compounds 8-21. As the first step, a hydrazone type intermediate is formed and a Michael type addition of its amino group provides the pyrazoline ring. According to previous experiences, ${ }^{32}$ the hydrazones of the $\alpha, \beta$-unsaturated ketones are so labile that they cannot be isolated. ${ }^{32}$ In our present study, no such an intermediate could be detected or isolated. This mechanism is in accordance with the fact that in the course of the ring closure there is no stereoselection if acetic acid is used and the trans- and cis-diastereomers are obtained as 1:1 mixtures (Table 1). In case propionic acid was used as solvent, a minimum stereoselection was observed (Tabel 1).

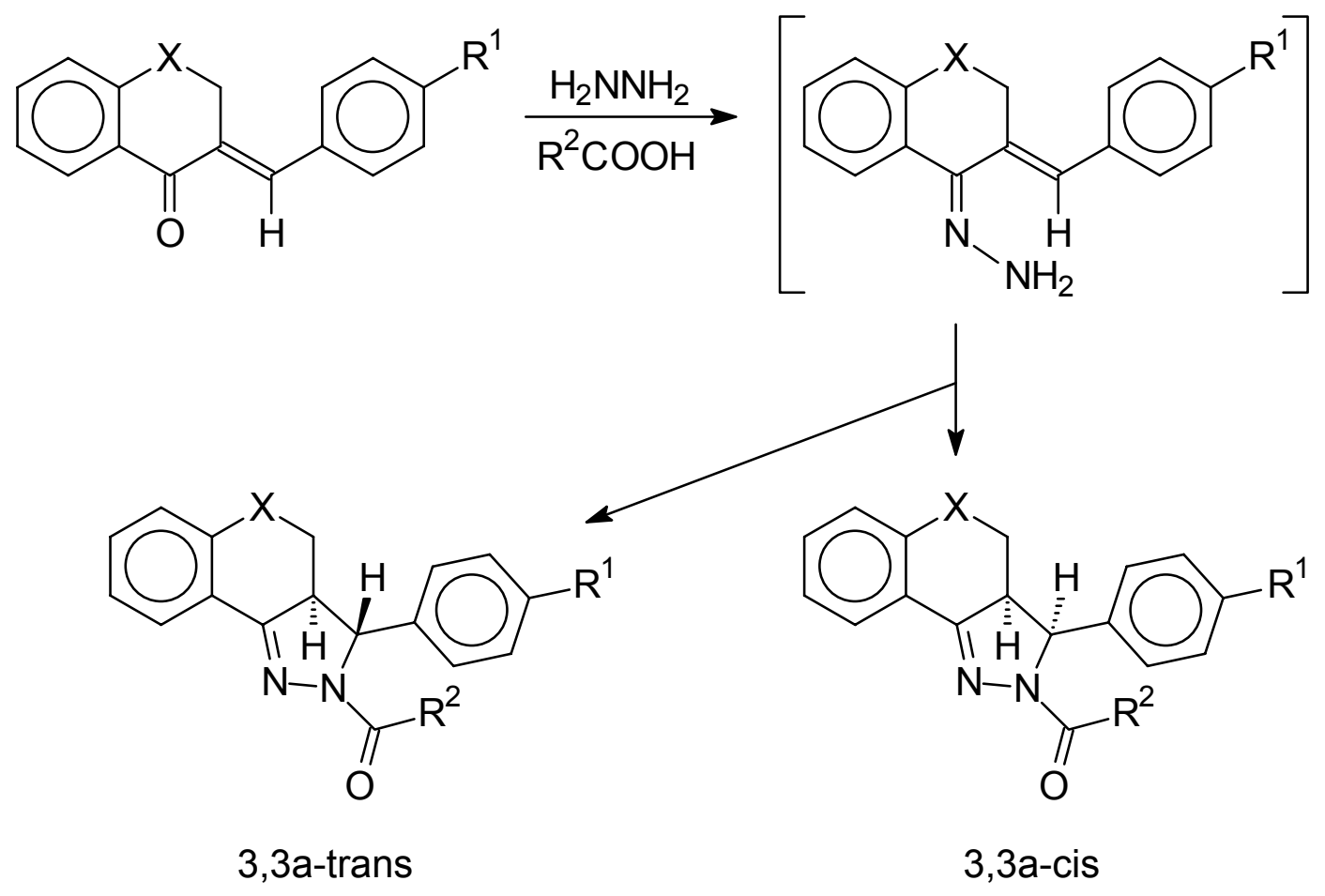

\section{Scheme 2}

In summary, it can be concluded that we have succeeded to prepare pure 3,3a-trans- and 3,3a-cis-diastereomers of tricyclic fused pyrazolines by the reaction of 3-arylidenechromanones and 3-arylidene-1-thiochromanones with hydrazine hydrate in hot acetic acid or propionic acid as solvents followed by column chromatographic separation. This made possible an unambiguous characterization of these pyrazoline isomers by microanalyses and spectroscopic measurements. 


\section{Experimental Section}

General Procedures. Melting points were determined on a Kofler hot-stage apparatus and are uncorrected. ${ }^{1} \mathrm{H}$ - and ${ }^{13} \mathrm{C}$-NMR spectra were measured with a Bruker WP $200 \mathrm{SY}$ spectrometer at $200 / 50 \mathrm{MHz}$ in $\mathrm{CDCl}_{3}$ (internal standard TMS, $\delta=0.0 \mathrm{ppm}$ ) at ambient temperature (ca 20 ${ }^{\circ} \mathrm{C}$ ). The selective transient NOE experiments were performed using double pulsed field gradient spin-echo exitation (DPFGSE-NOE ${ }^{31}$ ) on a Bruker Avance DRX 500 spectrometer equipped with z-axis gradient at ambient temperature. The IR spectra were obtained in $\mathrm{KBr}$ discs with a Perkin-Elmer 16 PC instrument. Mass spectra were recorded on a VG Trio-2 apparatus. Elemental analyses (C, H, N) were measured in-house with a Carlo Erba 1106 EA instrument. TLC was performed on Kieselgel $60 \mathrm{~F}_{254}$ (Merck) layer using toluene:ethyl acetate $(4: 1 \mathrm{v} / \mathrm{v})$ and hexane:acetone (7:3 v/v) as eluents. Starting materials 1-7 were synthesized according to known prucedures. $^{26,27}$

\section{General procedure for the preparation of compounds 8-21}

A mixture of the appropriate exocyclic $\alpha, \beta$-unsaturated ketone (1-7, 10.0 mmoles), hydrazine hydrate $(50.0$ mmoles $)$ and acetic acid $(40 \mathrm{~mL})$ or propionic acid $(40 \mathrm{~mL})$ was heated at reflux for $5 \mathrm{~h}$, then poured into water. The precipitate was separated by filtration, washed with water and dried to obtain compounds 8-21 (Scheme 1 and Table 1). The trans- and cis-diastereomers were separated by column chromatography on Kieselgel 60 columns using toluene:ethyl acetate $(4: 1 \mathrm{v} / \mathrm{v})$ as eluent.

trans-2,3,3a,4-Tetrahydro-2-acetyl-3-phenyl[1]benzopyrano[4,3-c]pyrazole

(trans-8).

Prepared as white needles in 27\% yield, mp 161-162 ${ }^{\circ} \mathrm{C} ;{ }^{1} \mathrm{H}-\mathrm{NMR}(\delta): 2.16(3 \mathrm{H}, \mathrm{s}, \mathrm{Me}), 3.26$ (1H, m, 3a-H), $3.86(1 \mathrm{H}, \mathrm{dd}, \mathrm{J}=10.3,12.8 \mathrm{~Hz}, 4-\mathrm{H}), 4.32(1 \mathrm{H}, \mathrm{dd}, \mathrm{J}=5.9,10.3 \mathrm{~Hz}, 4-\mathrm{H}), 4.68$ $(1 \mathrm{H}, \mathrm{d}, \mathrm{J}=9.4 \mathrm{~Hz}, 3-\mathrm{H}), 6.58-7.52$ (m, 9 arom. H); ${ }^{13} \mathrm{C}-\mathrm{NMR}(\delta): 22.2,52.7,63.8,68.8,115.2$, $117.5,121.7,124.9,125.8,127.7,128.9,132.3,140.8,149.8,156.6,170.3$; IR $\left(\mathrm{cm}^{-1}\right): 1652$, 1614, 1471, 1409, 1228, 1084, 1032, 995, 847, 759, 707; MS (m/z): $292\left(\mathrm{M}^{+}, 56\right), 250$ (100), 173 (24), 104 (94); Anal. Calcd. for $\mathrm{C}_{18} \mathrm{H}_{16} \mathrm{~N}_{2} \mathrm{O}_{2}$ : C, 73.95; H, 5.52; N, 9.58. Found: C, 73.86; H, $5.47 ; \mathrm{N}, 9.69$.

cis-2,3,3a,4-Tetrahydro-2-acetyl-3-phenyl[1]benzopyrano[4,3-c]pyrazole (cis-8). Obtained as white needles in $29 \%$ yield, mp $194-195{ }^{\circ} \mathrm{C} ;{ }^{1} \mathrm{H}-\mathrm{NMR}(\delta): 2.16(3 \mathrm{H}, \mathrm{s}, \mathrm{Me}), 2.93(1 \mathrm{H}, \mathrm{t}, \mathrm{J}=12.3$ $\mathrm{Hz}, 4-\mathrm{H}), 3.52(1 \mathrm{H}, \mathrm{m}, 3 \mathrm{a}-\mathrm{H}), 3.82(1 \mathrm{H}, \mathrm{dd}, \mathrm{J}=5.9,10.7 \mathrm{~Hz}, 4-\mathrm{H}), 5.44(1 \mathrm{H}, \mathrm{d}, \mathrm{J}=11.1 \mathrm{~Hz}, 3-$ $\mathrm{H}), 6.50-7.58$ (m, 9 arom. H); ${ }^{13} \mathrm{C}-\mathrm{NMR}(\delta): 21.8,45.6,61.6,67.1,115.6,117.6,121.7,125.1$, $128.1,132.5,135.6,150.2,156.7,168.2$; IR $\left(\mathrm{cm}^{-1}\right): 1651,1606,1440,1223,998,771,705 ; \mathrm{MS}$ (m/z): $292\left(\mathrm{M}^{+}, 63\right), 250$ 100), 173 (42), 104 (72); Anal. Calcd. for $\mathrm{C}_{18} \mathrm{H}_{16} \mathrm{~N}_{2} \mathrm{O}_{2}$ : C, 73.95; H, 5.52; N, 9.58. Found: 73.88; H, 5.58; N, 9.64.

\section{trans-2,3,3a,4-Tetrahydro-2-aceytl-3-(4-methylphenyl)[1]benzopyrano[4,3-c]pyrazole}

(trans-9). Isolated as white plates in $26 \%$ yield, mp $125-126{ }^{\circ} \mathrm{C} ;{ }^{1} \mathrm{H}-\mathrm{NMR}(\delta): 2.32(3 \mathrm{H}, \mathrm{s}, \mathrm{Me})$, $2.40(3 \mathrm{H}, \mathrm{s}, \mathrm{Me}), 3.57(1 \mathrm{H}, \mathrm{m}, 3 \mathrm{a}-\mathrm{H}), 4.17(1 \mathrm{H}, \mathrm{dd}, \mathrm{J}=10.5,12.8 \mathrm{~Hz}, 4-\mathrm{H}), 4.62(1 \mathrm{H}, \mathrm{dd}, \mathrm{J}=$ 5.8, $10.5 \mathrm{~Hz}, 4-\mathrm{H}), 4.98(1 \mathrm{H}, \mathrm{d}, \mathrm{J}=9.5 \mathrm{~Hz}, 3-\mathrm{H}), 6.90-7.84$ (m, 8 arom. H); ${ }^{13} \mathrm{C}-\mathrm{NMR}(\delta): 21.0$, 22.2, 52.7, 63.6, 68.8, 115.2, 117.4, 121.6, 124.9, 125.8, 129.6, 132.2, 137.4, 137.9, 149.8, 156.6, 
170.2; IR $\left(\mathrm{cm}^{-1}\right): 1667,1610,1472,1413,1332,1226,1120,998,826,759,653 ; \mathrm{MS}(\mathrm{m} / \mathrm{z}): 306$ $\left(\mathrm{M}^{+}, 72\right), 264$ (100), 173 (29), 118 (90); Anal. Calcd. for $\mathrm{C}_{19} \mathrm{H}_{18} \mathrm{~N}_{2} \mathrm{O}_{2}$ : C, 74.49; H, 5.92; N, 9.14. Found: C, 74.56; H, 5.87; N, 9.21.

cis-2,3,3a,4-Tetrahydro-2-acetyl-3-(4-methylphenyl)[1]benzopyrano[4,3-c]pyrazole (cis-9). Isolated as white plates in $21 \%$ yield, mp 146-147 ${ }^{\circ} \mathrm{C} ;{ }^{1} \mathrm{H}-\mathrm{NMR}(\delta): 2.30(3 \mathrm{H}, \mathrm{s}, \mathrm{Me}), 2.46(3 \mathrm{H}$, s, Me), $3.31(1 \mathrm{H}, \mathrm{dd}, \mathrm{J}=10.6,13.2 \mathrm{~Hz}, 4-\mathrm{H}), 3.85(1 \mathrm{H}, \mathrm{m}, 3 \mathrm{a}-\mathrm{H}), 4.16(1 \mathrm{H}, \mathrm{dd}, \mathrm{J}=5.8,10.6 \mathrm{~Hz}$, 4-H), 5.74 (1H, d, J = 11.1 Hz, 3-H), 6.84-7.89 (m, 8 arom. H); ${ }^{13} \mathrm{C}-\mathrm{NMR}(\delta): 20.9,21.8,45.4$, $61.4,67.2$, 115.7, 117.5, 121.6, 125.1, 125.4, 128.2, 129.0, 129.6, 132.4, 132.6, 137.8, 150.3, 156.7, 168.2; IR $\left(\mathrm{cm}^{-1}\right): 1659,1609,1473,1226,1002,825,781 ; \mathrm{MS}(\mathrm{m} / \mathrm{z}): 306\left(\mathrm{M}^{+}, 76\right), 264$ (100), 173 (31), 118 (79); Anal. Calcd. for $\mathrm{C}_{19} \mathrm{H}_{18} \mathrm{~N}_{2} \mathrm{O}_{2}$ : C, 74.49; H, 5.92; N, 9.14. Found: C, $74.41 ; \mathrm{H}, 5.96 ; 9.06$.

trans-2,3,3a,4-Tetrahydro-2-acetyl-3(4-methoxyphenyl)[1]benzopyrano[4,3-c]pyrazole (trans-10). Obtained as pale yellow needles in 29\% yield, mp 148-149 ${ }^{\circ} \mathrm{C} ;{ }^{1} \mathrm{H}-\mathrm{NMR}(\delta): 2.39$ (3H, s, Me), 3.59 (1H, m, 3a-H), 3.80 (3H, s, MeO), 4.18 (1H, t, J = $10.3 \mathrm{~Hz}, 4-\mathrm{H}), 4.64$ (1H, dd, $\mathrm{J}=5.9,10.1 \mathrm{~Hz}, 4-\mathrm{H}), 4.96(1 \mathrm{H}, \mathrm{d}, \mathrm{J}=9.5 \mathrm{~Hz}, 3-\mathrm{H}), 6.87-7.82(\mathrm{~m}, 8$ arom. $\mathrm{H}) ;{ }^{13} \mathrm{C}-\mathrm{NMR}(\delta)$ : 22,3, 52.7, 55.2, 63.4, 68.8, 114.3, 117.5, 121.7, 124.9, 127.2, 132.2, 132.9, 149.8, 156.7, 159.1, 170.3; IR ( $\left.\mathrm{cm}^{-1}\right): 1664,1610,1514,1413,1333,1248,1176,1033,999,832,763 . ; \mathrm{MS}(\mathrm{m} / \mathrm{z})$ : $322\left(\mathrm{M}^{+}, 56\right), 280$ (65), 146 (39), 134 (100); Anal. Calcd. for $\mathrm{C}_{19} \mathrm{H}_{18} \mathrm{~N}_{2} \mathrm{O}_{3}$ : C, 70.79; H, 5.63; N, 8.69. Found: C, 70.87; H, 5.69; N, 8.61.

cis-2,3,3a,4-Tetrahydro-2-acetyl-3-(methoxyphenyl)[1]benzopyrano[4,3-c]pyrazole (cis-10). Prepared as white plates in $25 \%$ yield, mp $177-178{ }^{\circ} \mathrm{C} ;{ }^{1} \mathrm{H}-\mathrm{NMR}(\delta): 2.46(3 \mathrm{H}, \mathrm{s}, \mathrm{Me}), 3.34(1 \mathrm{H}$, $\mathrm{t}, \mathrm{J}=10.5 \mathrm{~Hz}, 4-\mathrm{H}), 3.76(3 \mathrm{H}, \mathrm{s}, \mathrm{MeO}), 3.85(1 \mathrm{H}, \mathrm{m}, 3 \mathrm{a}-\mathrm{H}), 4.19$ (1H, dd, J = 5.8, $10.6 \mathrm{~Hz}, 4-\mathrm{H})$, $5.72(1 \mathrm{H}, \mathrm{d}, \mathrm{J}=11.0 \mathrm{~Hz}, 3-\mathrm{H}), 6.81-7.92$ (m, 8 arom. H); ${ }^{13} \mathrm{C}-\mathrm{NMR}(\delta): 21.7,45.6,55.2,61.1$, $67.2,114.4,115.6,117.6,121.6,125.1,126.7,127.8,132.4,150.3,156.7,159.4,168.2$; IR $\left(\mathrm{cm}^{-}\right.$ $\left.{ }^{1}\right): 1664,1611,1514,1472,1413,1248,1177,1033,999,832,763 ; \mathrm{MS}(\mathrm{m} / \mathrm{z}): 322\left(\mathrm{M}^{+}, 48\right), 280$ (52), 146 (31), 134 (100); Anal. Calcd. for $\mathrm{C}_{19} \mathrm{H}_{18} \mathrm{~N}_{2} \mathrm{O}_{3}$ : C, 70.79; H, 5.63; N, 8.69. Found: C, $70.70 ; \mathrm{H}, 5.57 ; \mathrm{N}, 8.76$.

trans-2,3,3a,4-Tetrahydro-2-acetyl-3-(4-chlorophenyl)[1]benzopyrano[4,3-c]pyrazole (trans-11). Prepared as yellow needles in $27 \%$ yield, mp 185-186 ${ }^{\circ} \mathrm{C} ;{ }^{1} \mathrm{H}-\mathrm{NMR}(\delta): 2.39(3 \mathrm{H}, \mathrm{s}$, Me), 3.52 (1H, m, 3a-H), $4.12(1 \mathrm{H}, \mathrm{dd}, \mathrm{J}=10.3,12.7 \mathrm{~Hz}, 4-\mathrm{H}), 4.60$ (1H, dd, J = 5.8, $10.3 \mathrm{~Hz}, 4-$ H), $4.94(1 \mathrm{H}, \mathrm{d}, \mathrm{J}=9.6 \mathrm{~Hz}, 3-\mathrm{H}), 6.88-7.80$ (m, 8 arom. H); ${ }^{13} \mathrm{C}-\mathrm{NMR}(\delta): 22.2,52.6,63.3,68.7$, $117.5,121.8,124.9,127.3,129.2,132.4,133.6,139.4,149.8,156.6,170.4 ;$ IR $\left(\mathrm{cm}^{-1}\right): 1668$, 1615, 1471, 1409, 1337, 1233, 1089, 1039, 847, 760, 542; MS (m/z): $326\left(\mathrm{M}^{+}, 57\right), 284$ (100), 173 (28), 130 (54); Anal. Calcd. for $\mathrm{C}_{18} \mathrm{H}_{15} \mathrm{ClN}_{2} \mathrm{O}_{2}$ : C, 66.16; H, 4.63; N, 8.57. Found: C, 66.25, $\mathrm{H}, 4.70 ; \mathrm{N}, 8.46$.

cis-2,3,3a,4-Tetrahydro-2-acetyl-3-(4-chlorophenyl)[1]benzopyrano[4,3-c]pyrazole (cis-11). Prepared as yellow plates in 21\% yield, mp 181-182 ${ }^{\circ} \mathrm{C} ;{ }^{1} \mathrm{H}-\mathrm{NMR}(\delta): 2.46(3 \mathrm{H}, \mathrm{s}, \mathrm{Me}), 3.32$ $(1 \mathrm{H}, \mathrm{dd}, \mathrm{J}=10.7,13.0 \mathrm{~Hz}, 4-\mathrm{H}), 3.84(1 \mathrm{H}, \mathrm{m}, 3 \mathrm{a}-\mathrm{H}), 4.13(1 \mathrm{H}, \mathrm{dd}, \mathrm{J}=5.7,10.7 \mathrm{~Hz}, 4-\mathrm{H}), 5.72$ $(1 \mathrm{H}, \mathrm{d}, \mathrm{J}=11.2 \mathrm{~Hz}, 3-\mathrm{H}), 6.88-7.92$ (m, 8 arom. H); ${ }^{13} \mathrm{C}-\mathrm{NMR}(\delta): 21.8,45.5,60.9,67.0,115.4$, $117.6,121.8,125.1,127.0,129.2,132.6,134.1,134.2,150.3,156.7,168.2$; IR $\left(\mathrm{cm}^{-1}\right): 1664$, 
1607, 1486, 1412, 1336, 1222, 1089, 998, 830, 764, 549; MS (m/z): $326\left(\mathrm{M}^{+}, 76\right), 284(100)$, 173 (41), 130 (53); Anal. Calcd. for $\mathrm{C}_{18} \mathrm{H}_{15} \mathrm{ClN}_{2} \mathrm{O}_{2}$ : C, 66.16; H, 4.63; N, 8.57. Found: C, 66.08; $\mathrm{H}, 4.57 ; \mathrm{N}, 8.64$.

trans-2,3,3a,4-Tetrahydro-3-phenyl-2-propionyl[1]benzopyrano[4,3-c]pyrazole (trans-12). Isolated as white needles in $33 \%$ yield, mp $172-173{ }^{\circ} \mathrm{C} ;{ }^{1} \mathrm{H}-\mathrm{NMR}(\delta): 1.18(3 \mathrm{H}, \mathrm{t}, \mathrm{J}=7.6 \mathrm{~Hz}$, $\left.\mathrm{CH}_{2} \mathrm{CH}_{3}\right), 2.49\left(2 \mathrm{H}, \mathrm{q}, \mathrm{J}=7.6 \mathrm{~Hz}, \mathrm{CH}_{2} \mathrm{CH}_{3}\right), 3.24(1 \mathrm{H}, \mathrm{m}, 3 \mathrm{a}-\mathrm{H}), 3.82(1 \mathrm{H}, \mathrm{dd}, \mathrm{J}=10.2,12.7$ $\mathrm{Hz}, 4-\mathrm{H}), 4.30$ (1H, dd, J = 5.9, $10.2 \mathrm{~Hz}, 4-\mathrm{H}), 4.68(1 \mathrm{H}, \mathrm{d}, \mathrm{J}=9.5 \mathrm{~Hz}, 3-\mathrm{H}), 6.57-7.50$ (m, 9 arom. H); ${ }^{13} \mathrm{C}-\mathrm{NMR}(\delta): 8.7,27.7,52.4,64.0,68.8,115.3,117.4,121.6,124.9,125.8,127.7$, 128.9, 132.2, 141.0, 149.6, 156.6, 173.7; IR( $\left.\mathrm{cm}^{-1}\right): 1662,1609,1426,1287,1232,1119,1048$, 1005, 847, 754, 706; MS (m/z): $306\left(\mathrm{M}^{+}, 31\right), 250$ (100), 173 (20), 104 (60); Anal. Calcd. for $\mathrm{C}_{19} \mathrm{H}_{18} \mathrm{~N}_{2} \mathrm{O}_{2}$ : C, 74.49; H, 5.92; N, 9.14. Found: C, 74.58; H, 5.97; N, 9.24.

cis-2,3,3a,4-Tetrahydro-3-phenyl-2-propionyl[1]benzopyrano[4,3-c]pyrazole (cis-12). Obtained as white needles in 25\% yield, mp 188-189 ${ }^{\circ} \mathrm{C} ;{ }^{1} \mathrm{H}-\mathrm{NMR}(\delta): 1.17(3 \mathrm{H}, \mathrm{t}, \mathrm{J}=7.5 \mathrm{~Hz}$, $\left.\mathrm{CH}_{2} \underline{\mathrm{C}}_{3}\right), 2.53\left(2 \mathrm{H}, \mathrm{q}, \mathrm{J}=7.5 \mathrm{~Hz}, \mathrm{C}_{2} \mathrm{CH}_{3}\right), 2.98(1 \mathrm{H}, \mathrm{dd}, \mathrm{J}=10.7,13.0 \mathrm{~Hz}, 4-\mathrm{H}), 3.54(1 \mathrm{H}, \mathrm{m}$, 3a-H), $3.83(1 \mathrm{H}, \mathrm{dd}, \mathrm{J}=5.8,10.7 \mathrm{~Hz}, 4-\mathrm{H}), 5.44(1 \mathrm{H}, \mathrm{d}, \mathrm{J}=11.1 \mathrm{~Hz}, 3-\mathrm{H}), 6.52-7.60$ (m, 9 arom. $\mathrm{H}) ;{ }^{13} \mathrm{C}-\mathrm{NMR}(\delta): 8.9,27.5,45.4,61.6,67.2,115.7,117.6,121.6,125.1,128.1,128.9,132.4$, 135.8, 150.0, 156.7, 171.7; IR (cm $\left.{ }^{-1}\right): 1660,1609,1457,1431,1223,1109$, 1032, 995, 850, 764; MS (m/z): $306\left(\mathrm{M}^{+}, 40\right), 250$ (100), 173 (31), 104 (45); Anal. Calcd. for $\mathrm{C}_{19} \mathrm{H}_{18} \mathrm{~N}_{2} \mathrm{O}_{2}$ : C, 74.49; H, 5.92, N, 9.14. Found: 74.38; H, 5.86, N, 9.22.

trans-2,3,3a,4-Tetrahydro-3-(4-methylphenyl)[1]benzopyrano[4,3-c]pyrazole (trans-13). Prepared as white plates in 30\% yield, mp 148-149 ${ }^{\circ} \mathrm{C} ;{ }^{1} \mathrm{H}-\mathrm{NMR}(\delta): 1.16(3 \mathrm{H}, \mathrm{t}, \mathrm{J}=7.3 \mathrm{~Hz}$, $\left.\mathrm{CH}_{2} \underline{\mathrm{C}}_{3}\right), 2.33(3 \mathrm{H}, \mathrm{s}, \mathrm{Me}), 2.80\left(2 \mathrm{H}, \mathrm{q}, \mathrm{J}=7.3 \mathrm{~Hz}, \mathrm{CH}_{2} \mathrm{CH}_{3}\right), 3.57$ (1H, m, 3a-H), 4.15 (1H, dd, $\mathrm{J}=10.2,12.8 \mathrm{~Hz}, 4-\mathrm{H}), 4.62(1 \mathrm{H}, \mathrm{dd}, \mathrm{J}=5.8,10.2 \mathrm{~Hz}, 4-\mathrm{H}), 4.96(1 \mathrm{H}, \mathrm{d}, 9.6 \mathrm{~Hz}, 3-\mathrm{H}), 6.90-$ 7.82 (m, 8 arom. H); ${ }^{13} \mathrm{C}-\mathrm{NMR}(\delta): 8.7,21.0,21.7,52.4,63.8,68.8,115.3,117.4,121.6,124.9$, $125.9,129.6,132.2,137.4,138.0,149.5,156.6,173.7$; IR $\left(\mathrm{cm}^{-1}\right): 1672,1614,1473,1414,1285$, 1230, 1046, 989, 817, 758; MS (m/z): $320\left(\mathrm{M}^{+}, 40\right), 264$ (100), 173 (16), 118 (58); Anal. Calcd. for $\mathrm{C}_{20} \mathrm{H}_{20} \mathrm{~N}_{2} \mathrm{O}_{2}$ : C, 74.98; H, 6.29, N, 8.74. Found: C, 74.89; H, 6.34, N, 8.66.

cis-2,3,3a,4-Tetryhydro-3-(4-methylphenyl)-2-propionyl[1]benzopyrano[4,3-c]pyrazole (cis13). Prepared as white plates in $21 \%$ yield, mp 136-137 ${ }^{\circ} \mathrm{C} ;{ }^{1} \mathrm{H}-\mathrm{NMR}(\delta): 1.22(3 \mathrm{H}, \mathrm{t}, \mathrm{J}=7.6$ $\left.\mathrm{Hz}, \mathrm{CH}_{2} \underline{\mathrm{C}}_{3}\right), 2.31$ (3H, s, Me), $2.85\left(2 \mathrm{H}, \mathrm{q}, \mathrm{J}=7.6 \mathrm{~Hz}, \mathrm{C}_{2} \mathrm{CH}_{3}\right), 3.32(1 \mathrm{H}, \mathrm{dd}, \mathrm{J}=10.5,12.6$ $\mathrm{Hz}, 4-\mathrm{H}), 3.83(1 \mathrm{H}, \mathrm{m}, 3 \mathrm{a}-\mathrm{H}), 4.17(1 \mathrm{H}, \mathrm{dd}, \mathrm{J}=5.6,10.7 \mathrm{~Hz}, 4-\mathrm{H}), 5.71(1 \mathrm{H}, \mathrm{d}, \mathrm{J}=11.5 \mathrm{~Hz}, 3-$ $\mathrm{H})$, 6.87-7.91 (m, 8 arom. H); ${ }^{13} \mathrm{C}-\mathrm{NMR}(\delta): 8.9,21.0,27.5,45.3,61.5,67.2,117.5,121.6$, 125.4, 129.6, 132.3, 132.8, 137.8, 150.0, 156.7, 171.6; IR ( $\left.\mathrm{cm}^{-1}\right): 1672,1611,1473,1414,1285$, 1230, 1046, 999, 845, 758; MS (m/z): $320\left(\mathrm{M}^{+}, 38\right), 264$ (100), 173 (15), 118 (53); Anal. Calcd. for $\mathrm{C}_{20} \mathrm{H}_{20} \mathrm{~N}_{2} \mathrm{O}_{2}$ : C, 74.98; 6.29; N, 8.74. Found: C, 74.90; H, 6.23; N, 8.81.

trans-2,3,3a,4-Tetrahydro-3-(4-methoxyphenyl)-2-propionyl[1]benzopyrano[4,3-c]pyrazole (trans-14). Prepared as white plates in $27 \%$ yield, mp $146-147{ }^{\circ} \mathrm{C} ;{ }^{1} \mathrm{H}-\mathrm{NMR}(\delta): 1.19(3 \mathrm{H}, \mathrm{t}, \mathrm{J}=$ $\left.7.3 \mathrm{~Hz}, \mathrm{CH}_{2} \underline{\mathrm{C}}_{3}\right), 2.80$ (2H, q, J = 7.3 Hz, $\left.\underline{\mathrm{C}}_{2} \mathrm{CH}_{3}\right), 3.59$ (1H, m, 3a-H), 3.79 (3H, s, MeO), $4.18(1 \mathrm{H}, \mathrm{dd}, \mathrm{J}=10.6,12.9 \mathrm{~Hz}, 4-\mathrm{H}), 4.62(1 \mathrm{H}, \mathrm{dd}, \mathrm{J}=5.9,10.2 \mathrm{~Hz}, 4-\mathrm{H}), 4.98(1 \mathrm{H}, \mathrm{d}, \mathrm{J}=9.5$ $\mathrm{Hz}, 3-\mathrm{H}), 6.87-7.82$ (m, 8 arom. H); ${ }^{13} \mathrm{C}-\mathrm{NMR}(\delta): 8.7,27.7,52.4,55.2,63.5,68.8,114.3,115.3$, 
117.4, 121.6, 124.9, 127.2, 132.2, 133.0, 149.6, 156.6, 159.1, 173.8; IR (cm $\left.{ }^{-1}\right): 1664,1611,1514$, 1458, 1417, 1288, 1248, 1178, 1033, 832, 764; MS (m/z): $336\left(\mathrm{M}^{+}, 42\right), 280$ (100), 146 (31), 134 (90); Anal. Calcd. for $\mathrm{C}_{20} \mathrm{H}_{20} \mathrm{~N}_{2} \mathrm{O}_{3}$ : C, 71.41; H, 5.99; N, 8.32. Found: C, 71.50; H, 5.93; N, 8.40. cis-2,3,3a,4-Tetrahydro-3-(4-methoxyphenyl)-2-propionyl[1]benzopyrano[4,3-c]pyrazole (cis-14). Isolated as white plates in $27 \%$ yield, mp 132-133 ${ }^{\circ} \mathrm{C} ;{ }^{1} \mathrm{H}-\mathrm{NMR}(\delta): 1.22(3 \mathrm{H}, \mathrm{t}, \mathrm{J}=7.8$ $\left.\mathrm{Hz}, \mathrm{CH}_{2} \mathrm{CH}_{3}\right), 2.85\left(2 \mathrm{H}, \mathrm{q}, \mathrm{J}=7.8 \mathrm{~Hz}, \mathrm{CH}_{2} \mathrm{CH}_{3}\right), 3.37(1 \mathrm{H}, \mathrm{dd}, \mathrm{J}=10.7,12.8 \mathrm{~Hz}, 4-\mathrm{H}), 3.75$ $(3 \mathrm{H}, \mathrm{s}, \mathrm{MeO}), 3.83(1 \mathrm{H}, \mathrm{m}, 3 \mathrm{a}-\mathrm{H}), 4.16(1 \mathrm{H}, \mathrm{dd}, \mathrm{J}=5.4,10.7 \mathrm{~Hz}, 4-\mathrm{H}), 5.73(1 \mathrm{H}, \mathrm{d}, \mathrm{J}=11.3 \mathrm{~Hz}$, 3-H), 6.80-7.92 (m, 8 arom. H); ${ }^{13} \mathrm{C}-\mathrm{NMR}(\delta): 8.9,27.5,45.4,55.2,61.2,67.2,115.7,117.6$, 121.6, 125.0, 126.7, 127.9, 128.0, 132.3, 150.0, 156.7, 159.3, 171.6; IR (cm $\left.{ }^{-1}\right): 1664,1610,1514$, 1458, 1417, 1288, 1248, 1178, 1033, 832, 765; MS (m/z): $336\left(\mathrm{M}^{+}, 34\right), 280(100), 146$ (32), 134 (75); Anal. Calcd. for $\mathrm{C}_{20} \mathrm{H}_{20} \mathrm{~N}_{2} \mathrm{O}_{3}$ : C, 71.41; H, 5.99; N, 8.32. Found: C, 71.43; H, 5.94; N, 8.26. trans-2,3,3a,4-Tetrahydro-3-(4-chlorophenyl)-2-propionyl[1]benzopyrano[4,3-c]pyrazole (trans-15). Obtained as yellow needles in 26\% yield, mp 198-199 ${ }^{\circ} \mathrm{C} ;{ }^{1} \mathrm{H}-\mathrm{NMR}(\delta): 1.20(3 \mathrm{H}, \mathrm{t}, \mathrm{J}$ $\left.=7.5 \mathrm{~Hz}, \mathrm{CH}_{2} \underline{\mathrm{CH}}_{3}\right), 2.80\left(2 \mathrm{H}, \mathrm{q}, \mathrm{J}=7.5 \mathrm{~Hz}, \mathrm{CH}_{2} \mathrm{CH}_{3}\right), 3.50(1 \mathrm{H}, \mathrm{m}, 3 \mathrm{a}-\mathrm{H}), 4.16(1 \mathrm{H}, \mathrm{dd}, 10.3$, $12.7 \mathrm{~Hz}, 4-\mathrm{H}), 4.60(1 \mathrm{H}, \mathrm{dd}, \mathrm{J}=5.9,10.3 \mathrm{~Hz}, 4-\mathrm{H}), 4.96(1 \mathrm{H}, \mathrm{d}, \mathrm{J}=9.7 \mathrm{~Hz}, 3-\mathrm{H}), 6.88-7.82(\mathrm{~m}$, 8 arom. H); ${ }^{13} \mathrm{C}-\mathrm{NMR}(\delta): 8.7,27.7,52.3,63.5,68.7,115.1,117.5,121.8,124.9,127.9,129.2$, 132.4, 133.6, 139.5, 149.6, 156.6, 173.9; IR $\left(\mathrm{cm}^{-1}\right): 1670,1615,1492,1473,1411,1284,1090$, 1046, 1013, 846, 757, 541; MS (m/z): $340\left(\mathrm{M}^{+}, 34\right), 284$ (100), 173 (22), 130 (36); Anal. Calcd. for $\mathrm{C}_{19} \mathrm{H}_{17} \mathrm{ClN}_{2} \mathrm{O}_{2}$ : C, 66.96; H, 5.03; N, 8.21. Found: C, 66.87; H, 5.09; N, 8.30.

cis-2,3,3a,4-Tetrahydro-3-(4-chlorophenyl)-2-propionyl[1]benzopyrano[4,3-c]pyrazole (cis15). Prepared as yellow plates in $28 \%$ yield, mp 185-186 ${ }^{\circ} \mathrm{C} ;{ }^{1} \mathrm{H}-\mathrm{NMR}(\delta): 1.22(3 \mathrm{H}, \mathrm{t}, \mathrm{J}=7.6$ $\left.\mathrm{Hz}, \mathrm{CH}_{2} \mathrm{CH}_{3}\right), 2.84\left(2 \mathrm{H}, \mathrm{q}, \mathrm{J}=7.6 \mathrm{~Hz}, \mathrm{CH}_{2} \mathrm{CH}_{3}\right), 3.29(1 \mathrm{H}, \mathrm{dd}, \mathrm{J}=10.8,11.9 \mathrm{~Hz}, 4-\mathrm{H}), 3.83$ $(1 \mathrm{H}, \mathrm{m}, 3 \mathrm{a}-\mathrm{H}), 4.16(1 \mathrm{H}, \mathrm{dd}, \mathrm{J}=5.7,10.8 \mathrm{~Hz}, 4-\mathrm{H}), 5.72(1 \mathrm{H}, \mathrm{d}, \mathrm{J}=11.2 \mathrm{~Hz}, 3-\mathrm{H}), 6.83-7.86(\mathrm{~m}$, 8 arom. $\mathrm{H}) ;{ }^{13} \mathrm{C}-\mathrm{NMR}(\delta): 8.9,27.5,45.3,61.0,67.0,115.5,117.6,121.7,125.1,127.0,129.2$, 132.6, 134.0, 134.4, 150.0, 156.7, 171.7; IR ( $\left.\mathrm{cm}^{-1}\right)$ : 1654, 1610, 1490, 1472, 1430, 1376, 1222 , 1091, 1008, 829, 764; MS (m/z): 340 (M+36), 284 (100), 173 (28), 130 (29); Anal. Calcd. for $\mathrm{C}_{19} \mathrm{H}_{17} \mathrm{ClN}_{2} \mathrm{O}_{2}$ : C, 66.96; H, 5.03, N, 8.21. Found: 66.88; H, 5.07; N, 8.14.

trans-2,3,3a,4-Tetrahydro-2-acetyl-3-phenyl[1]benzothiopyrano[4,3-c]pyrazole (trans-16). Isolated as pale yellow needles in $23 \%$ yield, mp 189-190 ${ }^{\circ} \mathrm{C} ;{ }^{1} \mathrm{H}-\mathrm{NMR}(\delta): 2.42(3 \mathrm{H}, \mathrm{s}, \mathrm{Me})$, $3.13(1 \mathrm{H}, \mathrm{dd}, \mathrm{J}=4.5,11.9 \mathrm{~Hz}, 4-\mathrm{H}), 3.38(1 \mathrm{H}, \mathrm{t}, \mathrm{J}=11.9 \mathrm{~Hz}, 4-\mathrm{H}), 3.60(1 \mathrm{H}, \mathrm{m}, 3 \mathrm{a}-\mathrm{H}), 5.08$ $(1 \mathrm{H}, \mathrm{d}, \mathrm{J}=8.3 \mathrm{~Hz}, 3-\mathrm{H}), 7.12-8.06$ (m, 9 arom. H); ${ }^{13} \mathrm{C}-\mathrm{NMR}(\delta): 22.0,30.3,54.1,67.3,125.0$, $125.3,125.7,126.4,126.7,127.8,129.0,130.4,135.8,140.9,151.5,169.9$; IR $\left(\mathrm{cm}^{-1}\right): 1656$, 1596, 1475, 1453, 1417, 1360, 1259, 1164, 1097, 1032, 964, 873, 759, 699, 652, 545; MS (m/z): $308\left(\mathrm{M}^{+}, 36\right), 266$ (32), 189 (16), 161 (100); Anal. Calcd. for $\mathrm{C}_{18} \mathrm{H}_{16} \mathrm{~N}_{2} \mathrm{OS}$ : C, 70.12; H, 5.23; N, 9.08. Found: 70.21; H, 5.17; 9.16.

cis-2,3,3a,4-Tetrahydro-2-acetyl-3-phenyl[1]benzothiopyrano[4,3-c]pyrazole

(cis-16).

Prepared as pale yellow needles in $28 \%$ yield, mp $217-218{ }^{\circ} \mathrm{C} ;{ }^{1} \mathrm{H}-\mathrm{NMR}(\delta): 2.43\left(2 \mathrm{H}, \mathrm{m}, 4-\mathrm{H}_{2}\right)$, 2.54 (3H, s, Me), 3.96 (1H, m, 3a-H), 5.72 (1H, d, J = $11.3 \mathrm{~Hz}, 3-\mathrm{H}), 7.04-8.18$ (m, 9 arom. H); ${ }^{13} \mathrm{C}-\mathrm{NMR}(\delta): 21.8,27.3,48.6,63.7,125.1,126.0,126.5,127.1,128.1,128.9,130.5,135.9$, 151.8, 169.6; IR (cm $\left.{ }^{-1}\right): 1651,1598,1452,1430,1336,1147,1045,965,872,770,702,549$; MS 
(m/z): $308\left(\mathrm{M}^{+}, 53\right), 266$ (46), 189 (58), 161 (100); Anal. Calcd. for $\mathrm{C}_{18} \mathrm{H}_{16} \mathrm{~N}_{2} \mathrm{OS}$ : C, 70.12; H, 5.23 ; N, 9.08. Found: C, 70.04; H, 5.29; N, 9.14.

\section{trans-2,3,3a,4-Tetrahydro-2-acetyl-3-(4-methylphenyl)[1]benzothiopyrano[4,3-c]pyrazole} (trans-17). Isolated as white needles in $33 \%$ yield, mp 92-93 ${ }^{\circ} \mathrm{C} ;{ }^{1} \mathrm{H}-\mathrm{NMR}(\delta): 2.37(3 \mathrm{H}, \mathrm{s}, \mathrm{Me})$, 2.39 (3H, s, Me), 3.04 (1H, dd, J = 4.7, $12.0 \mathrm{~Hz}, 4-\mathrm{H}), 3.59(1 \mathrm{H}, \mathrm{t}, \mathrm{J}=12.8 \mathrm{~Hz}, 4-\mathrm{H}), 3.56(1 \mathrm{H}$, m, 3a-H), $5.01(1 \mathrm{H}, \mathrm{d}, \mathrm{J}=8.4 \mathrm{~Hz}, 3-\mathrm{H}), 7.08-8.01$ (m, 8 arom. H); ${ }^{13} \mathrm{C}-\mathrm{NMR}(\delta): 20.9,21.8$, $30.1,53.9$, 66.9, 124.8, 125.2, 125.6, 126.1, 126.6, 129.5, 130.2, 135.7, 137.3, 137.9, 151.3, 169.6; IR $\left(\mathrm{cm}^{-1}\right): 1664,1608,1449,1413,1333,1143,958,873,778,550 ; \mathrm{MS}(\mathrm{m} / \mathrm{z}): 322\left(\mathrm{M}^{+}\right.$, 63), 280 (41), 189 (31), 161 (100); Anal. Cald. for $\mathrm{C}_{19} \mathrm{H}_{18} \mathrm{~N}_{2} \mathrm{OS}$ : C, 70.79; H, 5.63; N, 8.69. Found: C, 70.72; H, 5.59; N, 8.60.

cis-2,3,3a,4-Tetrahydro-2-acetyl-3-(4-methylphenyl)[1]benzothiopyrano[4,3-c]pyrazole (cis17). Obtained as white needles in $25 \%$ yield, mp 133-134 ${ }^{\circ} \mathrm{C} ;{ }^{1} \mathrm{H}-\mathrm{NMR}(\delta): 2.28(3 \mathrm{H}, \mathrm{s}, \mathrm{Me})$, $2.46(3 \mathrm{H}, \mathrm{s}, \mathrm{Me}), 2.51\left(2 \mathrm{H}, \mathrm{m}, 4-\mathrm{H}_{2}\right), 3.92(1 \mathrm{H}, \mathrm{m}, 3 \mathrm{a}-\mathrm{H}), 5.68(1 \mathrm{H}, \mathrm{d}, \mathrm{J}=11.3 \mathrm{~Hz}, 3-\mathrm{H}), 6.94-$ 8.13 (m, 8 arom. H); ${ }^{13} \mathrm{C}-\mathrm{NMR}(\delta): 21.0,21.8,27.3,48.4,63.5,125.0,125.9,126.4,127.1$, 129.6, 130.4, 135.9, 137.8, 151.8, 168.2; IR $\left(\mathrm{cm}^{-1}\right)$ : 1660, 1612, 1449, 1413, 1333, 1143, 1031, 957, 873, 805, 778; MS (m/z): $322\left(\mathrm{M}^{+}, 54\right), 280$ (40), 189 (30), 161 (100); Anal. Calcd. for $\mathrm{C}_{19} \mathrm{H}_{18} \mathrm{~N}_{2} \mathrm{OS}$ : C, 70.79; H, 5.63; N, 8.69. Found: C, 70.87; H, 5.69; N, 8.61.

trans-2,3,3a,4-Tetrahydro-2-acetyl-3-(4-methoxyphenyl)[1]benzothiopyrano[4,3-c]pyrazole (trans-18). Prepared as white plates in $27 \%$ yield, mp 132-133 ${ }^{\circ} \mathrm{C}$; ${ }^{1} \mathrm{H}-\mathrm{NMR}(\delta): 2.39(3 \mathrm{H}$, s, Me), 3.09 (1H, dd, J = 4.6, $12.0 \mathrm{~Hz}, 4-\mathrm{H}), 3.36$ (1H, t, J = $12.0 \mathrm{~Hz}, 4-\mathrm{H}), 3.61$ (1H, m, 3a-H), $3.80(3 \mathrm{H}, \mathrm{s}, \mathrm{MeO}), 5.04(1 \mathrm{H}, \mathrm{d}, \mathrm{J}=8.4 \mathrm{~Hz}, 3-\mathrm{H}), 6.88-8.02\left(\mathrm{~m}, 8\right.$ arom. H); ${ }^{13} \mathrm{C}-\mathrm{NMR}(\delta): 22.0$, $30.4,54.1,55.3,66.8,114.4,125.0,125.4,126.4,127.1,127.2,130.4,133.1,135.8,151.6,159.9$, 169.9; IR ( $\left.\mathrm{cm}^{-1}\right): 1660,1606,1510,1414,1334,1244,1174,1030,955,759,554 ; \mathrm{MS}(\mathrm{m} / \mathrm{z}): 338$ $\left(\mathrm{M}^{+}, 59\right), 296$ (32), 161 (75), 135 (100); Anal. Calcd. for $\mathrm{C}_{19} \mathrm{H}_{18} \mathrm{~N}_{2} \mathrm{O}_{2} \mathrm{~S}: \mathrm{C}, 67.44 ; \mathrm{H}, 5.36$; N, 8.27. Found: C, 67.52; H, 5.42; N. 8.34.

cis-2,3,3a,4-Tetrahydro-2-acetyl-3-(4-methoxyphenyl)[1]benzothiopyrano[4,3-c]pyrazole (cis-18). Obtained as white plates in 35\% yield, mp 101-102 ${ }^{\circ} \mathrm{C} ;{ }^{1} \mathrm{H}-\mathrm{NMR}(\delta): 2.36(2 \mathrm{H}, \mathrm{m}, 4-$ $\left.\mathrm{H}_{2}\right), 2.45(3 \mathrm{H}, \mathrm{s}, \mathrm{Me}), 3.77(3 \mathrm{H}, \mathrm{s}, \mathrm{MeO}), 3.92(1 \mathrm{H}, \mathrm{m}, 3 \mathrm{a}-\mathrm{H}), 5.68(1 \mathrm{H}, \mathrm{d}, \mathrm{J}=11.2 \mathrm{~Hz}, 3-\mathrm{H})$, 6.82-8.16 )m, 8 arom. H); ${ }^{13} \mathrm{C}-\mathrm{NMR}(\delta): 21.8,27.2$, 48.5, 55.1, 63.2, 114.3, 125.0, 125.9, 126.4, 127.2, 128.2, 129.0, 129.8, 130.4, 135.9, 151.8, 159.3, 168.1; IR $\left(\mathrm{cm}^{-1}\right): 1657,1612,1512,1411$, 1334, 1246, 1174, 1030, 956, 872, 822, 759; MS (m/z): $338\left(\mathrm{M}^{+}, 53\right), 296$ (30), 161 (78), 135 (100); Anal. Calcd. for $\mathrm{C}_{19} \mathrm{H}_{18} \mathrm{~N}_{2} \mathrm{O}_{2} \mathrm{~S}$ : C, 67.44; H, 5.36; N, 8.27. Found: 67.36; H, 5.31; N, 819.

trans-2,3,3a,4-Tetrahydro-3-phenyl-2-propionyl[1]benzothiopyrano[4,3-c]pyrazole (trans19). Isolated as white needles in $28 \%$ yield, mp $142-143{ }^{\circ} \mathrm{C} ;{ }^{1} \mathrm{H}-\mathrm{NMR}(\delta): 1.18(3 \mathrm{H}, \mathrm{t}, \mathrm{J}=7.5$ $\left.\mathrm{Hz}, \mathrm{CH}_{2} \underline{\mathrm{CH}}_{3}\right), 2.81\left(2 \mathrm{H}, \mathrm{q}, \mathrm{J}=7.5 \mathrm{~Hz}, \mathrm{CH}_{2} \mathrm{CH}_{3}\right), 3.10(1 \mathrm{H}, \mathrm{dd}, \mathrm{J}=4.6,11.9 \mathrm{~Hz}, 4-\mathrm{H}), 3.37(1 \mathrm{H}$, $\mathrm{t}, \mathrm{J}=11.9 \mathrm{~Hz}, 4-\mathrm{H}), 3.60(1 \mathrm{H}, \mathrm{m}, 3 \mathrm{a}-\mathrm{H}), 5.06$ (1H, d, J = 8.3 Hz, 3-H), 7.14-8.04 (m, 9 arom. H); ${ }^{13} \mathrm{C}-\mathrm{NMR}(\delta): 8.8,27.6,30.4,53.9,67.5,125.0,125.5,125.8,126.4,126.8,127.8,129.0,130.3$, 135.8, 141.1, 151.3, 173.4; IR ( $\left.\mathrm{cm}^{-1}\right): 1665,1596,1446,1412,1293,1257,1138,1023,965,864$, 761, 701, 547; MS (m/z): $322\left(\mathrm{M}^{+}, 27\right), 266$ (46), 189 (15), 161 (100); Anal. Calcd. for $\mathrm{C}_{19} \mathrm{H}_{18} \mathrm{~N}_{2} \mathrm{OS}$ : C, 70.79; H, 5.63; N, 8.68. Found: C, 70.87; H, 5.57; N, 8.76. 
cis-2,3,3a,4-Tetrahydro-3-phenyl-2-propionyl[1]benzothiopyrano[4,3-c]pyrazole (cis-19). Obtained as white needles in 23\% yield, mp 220-221 ${ }^{\circ} \mathrm{C} ;{ }^{1} \mathrm{H}-\mathrm{NMR}(\delta): 1.23(3 \mathrm{H}, \mathrm{t}, \mathrm{J}=7.5 \mathrm{~Hz}$, $\left.\mathrm{CH}_{2} \mathrm{CH}_{3}\right), 2.46\left(2 \mathrm{H}, \mathrm{m}, 4-\mathrm{H}_{2}\right), 2.88\left(2 \mathrm{H}, \mathrm{q}, \mathrm{J}=7.5 \mathrm{~Hz}, \mathrm{CH}_{2} \mathrm{CH}_{3}\right), 3.96(1 \mathrm{H}, \mathrm{m}, 3 \mathrm{a}-\mathrm{H}), 5.68(1 \mathrm{H}$, $\mathrm{d}, \mathrm{J}=11.3 \mathrm{~Hz}, 3-\mathrm{H}), 7.06-8.18$ (m, 9 arom. H); ${ }^{13} \mathrm{C}-\mathrm{NMR}(\delta): 8.9,27.3,27.4,48.3,63.8,125.1$, $125.8,126.0,126.5,127.1,128.1,128.9,130.4,135.9,136.2,151.6,171.7$; IR $\left(\mathrm{cm}^{-1}\right): 1660$, 1593, 1449, 1422, 1306, 1265, 1146, 1080, 862, 767, 701, 558; MS (m/z): $322\left(\mathrm{M}^{+}, 40\right), 266$ (71), 189 (47), 161 (100); Anal. Calcd. for $\mathrm{C}_{19} \mathrm{H}_{18} \mathrm{~N}_{2} \mathrm{OS}$ : C, 70.79; H, 5.63; N, 8.68. Found: $70.71 ; \mathrm{H}, 5.68 ; \mathrm{N}, 8.60$.

trans-2,3,3a,4-Tetrahydro-3-(4-methylphenyl)-2-propionyl[1] benzothiopyrano[4,3clpyrazole (trans-20). Prepared as white plates in $26 \%$ yield, mp $127-128{ }^{\circ} \mathrm{C} ;{ }^{1} \mathrm{H}-\mathrm{NMR}(\delta): 1.20$ $\left(3 \mathrm{H}, \mathrm{t}, \mathrm{J}=7.8 \mathrm{~Hz}, \mathrm{CH}_{2} \mathrm{CH}_{3}\right), 2.20(3 \mathrm{H}, \mathrm{s}, \mathrm{Me}), 2.80\left(2 \mathrm{H}, \mathrm{q}, \mathrm{J}=7.8 \mathrm{~Hz}, \mathrm{C}_{2} \mathrm{CH}_{3}\right), 3.08(1 \mathrm{H}, \mathrm{dd}, \mathrm{J}$ $=4.4,11.7 \mathrm{~Hz}, 4-\mathrm{H}), 3.34(1 \mathrm{H}, \mathrm{t}, \mathrm{J}=11.7 \mathrm{~Hz}, 4-\mathrm{H}), 3.59(1 \mathrm{H}, \mathrm{m}, 3 \mathrm{a}-\mathrm{H}), 5.04(1 \mathrm{H}, \mathrm{d}, \mathrm{J}=8.3 \mathrm{~Hz}$, 3-H), 7.10-8.04 (m, 8 arom. H); ${ }^{13} \mathrm{C}-\mathrm{NMR}(\delta): 8.7,21.0,27.5,30.3,53.9,67.3,124.9,125.7$, 126.3, 126.7, 129.6, 130.3, 135.8, 137.5, 138.1, 151.2, 173.3; IR $\left(\mathrm{cm}^{-1}\right): 1663,1602,1412,1354$, 1286, 1160, 1014, 867, 802, 754; MS (m/z): $336\left(\mathrm{M}^{+}, 27\right), 280$ (40), 189 (11), 161 (100); Anal. Calcd. for $\mathrm{C}_{20} \mathrm{H}_{20} \mathrm{~N}_{2} \mathrm{OS}$ : C, 71.41; H, 5.99; N, 8.32. Found: C, 71.52; H, 6.04; N, 8.26.

cis-2,3,3a,4-Tetrahydro-3-(4-methylphenyl)-2-propionyl[1]benzothiopyrano[4,3-c]pyrazole (cis-20). Isolated as white needles in $22 \%$ yield, mp 171-172 ${ }^{\circ} \mathrm{C} ;{ }^{1} \mathrm{H}-\mathrm{NMR}(\delta): 1.21$ (3H, t, J = $\left.7.9 \mathrm{~Hz}, \mathrm{CH}_{2} \mathrm{CH}_{3}\right), 2.19$ (3H, s, Me), $2.43\left(2 \mathrm{H}, \mathrm{m}, 4-\mathrm{H}_{2}\right), 2.87\left(2 \mathrm{H}, \mathrm{q}, \mathrm{J}=7.9 \mathrm{~Hz}, \mathrm{CH}_{2} \mathrm{CH}_{3}\right), 3.94$ $(1 \mathrm{H}, \mathrm{m}, 3 \mathrm{a}-\mathrm{H}), 5.69(1 \mathrm{H}, \mathrm{d}, \mathrm{J}=10.9 \mathrm{~Hz}, 3-\mathrm{H}), 6.97-8.18(\mathrm{~m}, 8$ arom. $\mathrm{H}) ;{ }^{13} \mathrm{C}-\mathrm{NMR}(\delta): 8.8,21.0$, $27.5,30.8,48.2$, 63.6, 125.0, 125.9, 126.4, 127.1, 129.6, 130.3, 133.1, 135.9, 137.8, 151.6, 171.7; IR ( $\left.\mathrm{cm}^{-1}\right): 1662,1608,1449,1419,1351,1304,1265,1146,1082,862,808,761,566$; MS (m/z): $336\left(\mathrm{M}^{+}, 59\right), 280$ (76), 189 (31), 161 (100); Anal. Calcd. for $\mathrm{C}_{20} \mathrm{H}_{20} \mathrm{~N}_{2} \mathrm{OS}$ : C, 71.41; H, 5.99; N, 8.32. Found: 71.37; H, 5.92; N, 8.41.

trans-2,3,3a,4-Tetrahydro-3-(4-methoxyphenyl)-2-propionyl[1]benzothiopyrano[4,3-

c]pyrazole (trans-21). Obtained as whte plates in 24\% yield, mp 156-157 ${ }^{\circ} \mathrm{C} ;{ }^{1} \mathrm{H}-\mathrm{NMR}(\delta): 1.19$ $\left(3 \mathrm{H}, \mathrm{t}, \mathrm{J}=7.7 \mathrm{~Hz}, \mathrm{CH}_{2} \underline{\mathrm{C}}_{3}\right), 2.80\left(2 \mathrm{H}, \mathrm{q}, \mathrm{J}=7.7 \mathrm{~Hz}, \mathrm{C}_{2} \mathrm{CH}_{3}\right), 3.10(1 \mathrm{H}, \mathrm{dd}, \mathrm{J}=4.5,11.8 \mathrm{~Hz}, 4-$ H), $3.34(1 \mathrm{H}, \mathrm{t}, \mathrm{J}=11.8 \mathrm{~Hz}, 4-\mathrm{H}), 3.59(1 \mathrm{H}, \mathrm{m}, 3 \mathrm{a}-\mathrm{H}), 3.82(3 \mathrm{H}, \mathrm{s}, \mathrm{MeO}), 5.04(1 \mathrm{H}, \mathrm{d}, \mathrm{J}=8.4$ $\mathrm{Hz}, 3-\mathrm{H}), 6.87-8.01$ (m, 8 arom. H); ${ }^{13} \mathrm{C}-\mathrm{NMR}(\delta): 8.8,27.6,30.3,53.9,55.2,67.1,114.4,125.0$, $126.4,126.8,127.2,130.3,133.3,151.3,159.2,173.5 ;$ IR $\left(\mathrm{cm}^{-1}\right): 1659,1612,1512,1446,1417$, 1248, 1176, 1035, 863, 761, 553; MS (m/z): $352\left(\mathrm{M}^{+}, 56\right), 296$ (53), 161 (74), 135 (100); Anal. Calcd. for $\mathrm{C}_{20} \mathrm{H}_{20} \mathrm{~N}_{2} \mathrm{O}_{2} \mathrm{~S}$ : C, 68.17; H, 5.72; N, 7.95. Found: C, 68.27; H, 5.66; N, 7.88.

cis-2,3,3a,4-Tetrahydro-3-(4-methoxyphenyl)-2-propionyl[1]benzothiopyrano[4,3-

c]pyrazole (cis-21). Prepared as white plates in $28 \%$ yield, mp $97-98{ }^{\circ} \mathrm{C} ;{ }^{1} \mathrm{H}-\mathrm{NMR}(\delta): 1.22(3 \mathrm{H}$, $\left.\mathrm{t}, \mathrm{J}=7.5 \mathrm{~Hz}, \mathrm{CH}_{2} \underline{\mathrm{C}}_{3}\right), 2.41\left(2 \mathrm{H}, \mathrm{m}, 4-\mathrm{H}_{2}\right), 2.84\left(2 \mathrm{H}, \mathrm{q}, \mathrm{J}=7.5 \mathrm{~Hz}, \mathrm{C}_{2} \mathrm{CH}_{3}\right), 3.77(3 \mathrm{H}, \mathrm{s}$, $\mathrm{MeO}), 3.93(1 \mathrm{H}, \mathrm{m}, 3 \mathrm{a}-\mathrm{H}), 5.67(1 \mathrm{H}, \mathrm{d}, \mathrm{J}=9.4 \mathrm{~Hz}, 3-\mathrm{H}), 6.81-8.14$ (m, 8 arom. H); ${ }^{13} \mathrm{C}-\mathrm{NMR}$ (ठ): 9.0, 27.5, 30.9, 48.4, 55.2, 63.4, 114.3, 125.1, 126.0, 126.4, 127.2, 128.4, 130.3, 135.9, 151.6, 159.4, 171.7; IR (cm $\left.{ }^{-1}\right): 1662,1608,1513,1447,1417,1249,1176,1034,862,759,555$; MS (m/z): $352\left(\mathrm{M}^{+}, 50\right), 296$ (57), 161 (71), 135 (100); Anal. Calcd. for $\mathrm{C}_{20} \mathrm{H}_{20} \mathrm{~N}_{2} \mathrm{O}_{2} \mathrm{~S}: \mathrm{C}, 68.17$; H, 5.72; N, 7.95. Found: C, 68.11; H, 5.79; N, 7.86. 


\section{Acknowledgements}

The present study was sponsored by the Hungarian National Research Fund (Grant No. OTKA T049468) for which our gratitude is expressed. Technical assistance of Mrs. M. Nagy is highly appreciated.

\section{References}

1. For Part 10, see: Lévai, A. Heterocycl. Commun. 2003, 9, 287.

2. Ramalingam, K.; Thyvelikakath, G. X.; Berlin, K. D.; Chesnut, R. W.; Brown, R. A.; Durham, N. N.; Ealick, S. E.; Van der Helm, D. J. Med. Chem. 1977, $20,847$.

3. Lombardino, J. G.; Otternes, I. G. J. Med. Chem. 1981, 24, 830.

4. Wrzeciono, U.; Pietkiewicz, K.; Krzysztofik, B.; Michalska, W.; Drozdowski, M. Pharmazie 1978, 33, 266.

5. Wiley, R. H., Ed. Pyrazoles, Pyrazolines, Pyrazolidines, Indazoles and Condensed Rings, In The Chemistry of Heterocyclic Compounds, Vol. 22, Weisberger, A., Ed., Interscience Publishers: New York, 1967; p 180.

6. Lévai, A. Khim. Geterotsikl. Soedin. 1997, 747.

7. Lévai, A. J. Heterocycl. Chem. 2002, 39, 1.

8. Turk, C. F. Ger. Offen. 1975, DE 2,520,171; Chem. Abstr. 1976, 84, 74261.

9. Krapcho, J.; Schwartz, J. US Patent 1976, 3,969,527; Chem. Abstr. 1976, 85, 177415.

10. Sangwan, N. K.; Rastogi, S. N. Indian J. Chem. 1981, 20B, 135.

11. Lóránd, T.; Szabó, D.; Földesi, A.; Párkányi, L.; Kálmán, A.; Neszmélyi, A. J. Chem. Soc., Perkin Trans. 1 1985, 481.

12. Lévai, A.; Szöllősy, Á.; Tóth, G. J. Chem. Research (S) 1985, 392.

13. Kamecki, J.; Perka, W.; Pijewska, L. Polish J. Chem. 1985, 59, 285.

14. El-Rayyes, N. R.; Al-Jawhary, A. J. Heterocycl. Chem. 1986, 23, 135.

15. Sangwan, N. K. J. Chem. Research (S) 1987, 22.

16. El-Rayyes, N. R.; Bahtiti, N. H. J. Heterocycl. Chem. 1989, 26, 209.

17. Tóth, G.; Szöllősy, Á.; Lóránd, T.; Kónya, T.; Szabó, D.; Földesi, A.; Lévai, A. J. Chem. Soc., Perkin Trans. 2 1989, 319.

18. Szöllősy, Á.; Tóth, G.; Lóránd, T.; Kónya, T.; Aradi, F.; Lévai, A. J. Chem. Soc., Perkin Trans. 2 1991, 489.

19. Faidallah, H. M.; Makki, M. S. I. J. Chin. Chem. Soc. 1994, 41, 585.

20. Lóránd, T.; Aradi, F.; Szöllősy, Á.; Tóth, G.; Kónya, T. Monatsh. Chem. 1996, 127, 971.

21. Lévai, A. J. Heterocycl. Chem. 1998, 35, 13.

22. Lévai, A. Heterocycl. Commun. 1999, 5, 151.

23. Mishriky, N.; Ibrahim, Y. A.; Girgis, A. S.; Fawzy, N. G. Pharmazie 1999, 54, 738.

24. Peesapati, V.; Sreelakshmi, P.; Anuradha, K. J. Chem. Research (S) 2001, 372. 
25. López, E. A. V.; Klimova, E. I.; Klimova, T.; Toledano, C. A.; Ramírez, L. R.; Toscano, R. A.; García, M. M. Synthesis 2004, 2471.

26. Lévai, A.; Schág, J. B. Pharmazie 1979, 34, 749.

27. Lévai, A.; Dinya, Z.; Schág, J. B.; Tóth, G.; Szöllősy, Á. Pharmazie 1981, 36, 465.

28. Lévai, A. J. Heterocycl. Chem. 2004, 41, 299.

29. Tóth, G.; Szöllősy, Á.; Lévai, A.; Kotovych, G. J. Chem. Soc., Perkin Trans. 2 1986, 1895.

30. Tóth, G.; Lévai, A.; Szöllősy, Á.; Duddeck, H. Tetrahedron 1993, 49, 863.

31. Stott, K.; Stonehouse, J.; Keeler, J.; Hwang, T-L.; Shaka, A. J. J. Am. Chem. Soc. 1995, 117, 4199.

32. Raiford, L. C.; Peterson, W. J. J. Org. Chem. 1936, 1, 544.

33. Aubagnac, J. L.; Elguero, J.; Jacquier, R. Bull. Soc. Chim. Fr. 1969, 3292.

34. Al-Farkh, Y. A.; Al-Hajjar, F. H.; Al-Shamali, F. S.; Hamoud, H. S. Chem. Pharm. Bull. 1979, 27, 257. 